\title{
A pellet tracking system for the PANDA experiment
}

\author{
A. Pyszniak · H. Calén • K. Fransson • C-J. Fridén • \\ E. Hellbeck • M. Jacewicz • T. Johansson • \\ P. Marciniewski $\cdot$ Z. Rudy
}

Received: 13 September 2013 / Accepted: 18 February 2014 / Published online: 20 March 2014

(C) The Author(s) 2014. This article is published with open access at Springerlink.com

\begin{abstract}
Frozen microspheres of hydrogen (pellets) will be one of the target types for the future hadron physics experiment PANDA at FAIR (GSI, Darmstadt, Germany). Pellets with a diameter of $25-\mu \mathrm{m}$ are generated about 3 meters above the interaction region, to which they travel with a velocity around $80 \mathrm{~m} / \mathrm{s}$ inside a narrow pipe. The interaction region is defined by the overlap of the pellet stream and the accelerator beam and has a size of a few millimeters. One would like to know the interaction point more precisely, to have better possibilities to reconstruct particle tracks and events e.g. in charmonium decay studies. One would also like to suppress background events that do not originate in a pellet, but e.g. may occur in rest gas, that is present in the beam pipe. A solution is provided by the presented pellet tracking system together with a target operation mode that provides one and only one pellet in the interaction region most of the time. The goal is to track individual pellets in order to know their position with a resolution of a few tenths of a millimeter at the time of an interaction. The system must also be highly efficient and provide tracking information for essentially all pellets that pass the interaction region. Presented results from the design studies show that the goals can be fulfilled by this solution.
\end{abstract}

Keywords Pellet · Pellet-tracking · Internal target

Proceedings of the 11th International Conference on Low Energy Antiproton Physics (LEAP 2013) held in Uppsala, Sweden, 10-15 June, 2013

A. Pyszniak $(\bowtie) \cdot$ Z. Rudy

Institute of Physics, Jagiellonian University, 30-059 Cracow, Poland

e-mail: andrzej.pyszniak@gmail.com

H. Calén · K. Fransson - E. Hellbeck · M. Jacewicz - T. Johansson · P. Marciniewski · A. Pyszniak Department of Physics and Astronomy, Uppsala University, 75120 Uppsala, Sweden

C-J. Fridén

The Svedberg Laboratory, Uppsala University, 75121 Uppsala, Sweden 


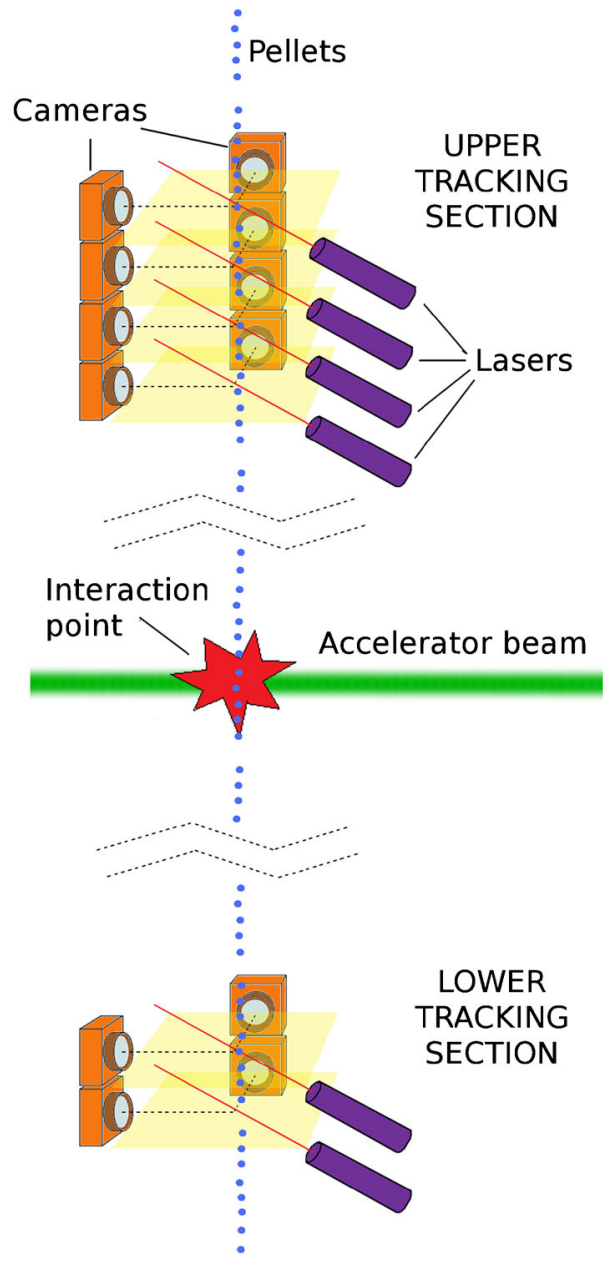

Fig. 1 Principle of operation of a pellet tracking system

\section{Introduction}

The tracking of pellets will be realized using lasers and fast line-scan (i.e. "one dimensional") CCD cameras. The cameras, placed at different levels along the pellet stream, measure pellet position and time of passage (Fig. 1). The measurement data from many cameras will be put together to identify and reconstruct the track of each pellet. Information about the pellet position in the interaction region at the time of an interaction will be used in the analysis of the experimental data.

\section{Design studies and preparation of a multi-camera system}

Tests and measurements with a prototype system of two synchronized cameras at the Uppsala Pellet Test Station (UPTS, Fig. 2) [1] are important for the design studies of the 

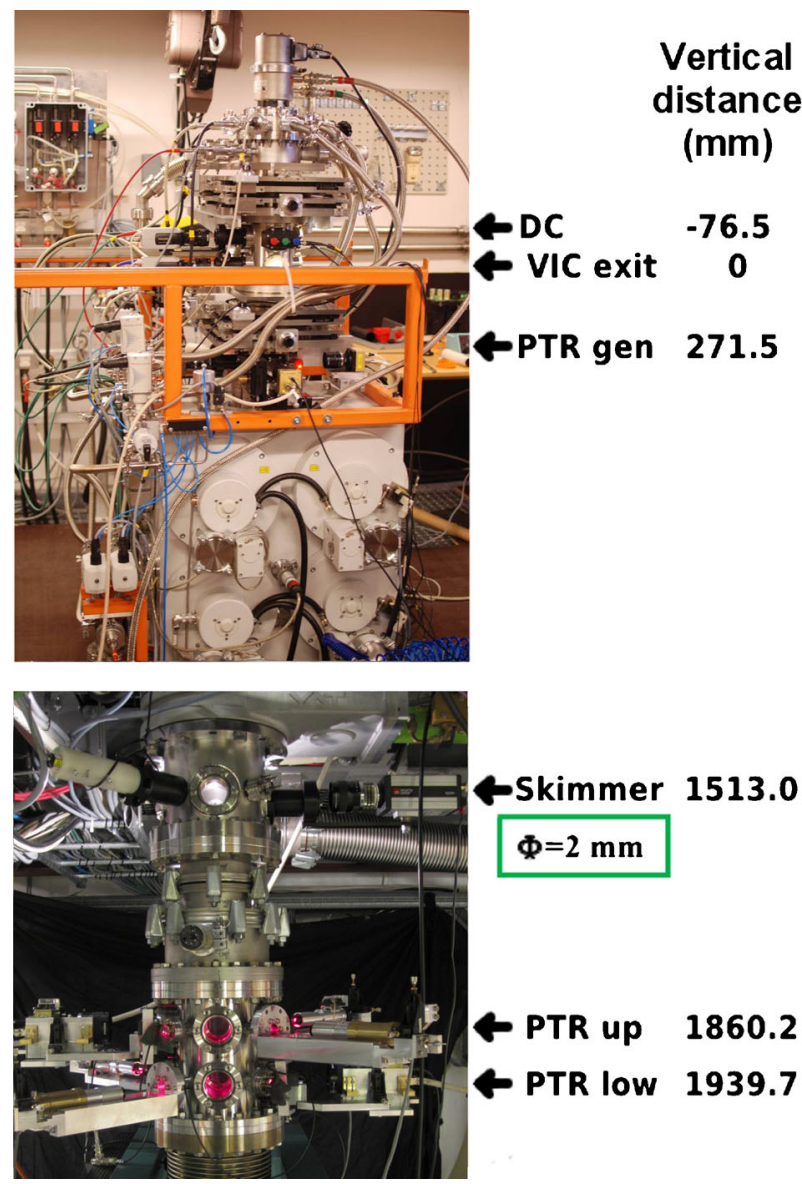

Fig. 2 The UPTS setup. Different pellet detection levels are indicated (see text)

tracking system. In the upper part where the pellets are generated, there are detection possibilities at the droplet chamber (DC), at the exit of the vacuum injection capillary (VIC) and above the turbo pump section (PTR gen). Below the pumps, in the lower part, there are detection possibilities just above the skimmer and at two levels in a special pellet tracking chamber. By measuring the pellet time of flight between different levels, the pellet velocities and the velocity spread $(\Delta v / v \approx 0.5 \%)$ have been measured. The position resolution obtained at the measurement levels has been determined to be $\approx 20 \mu \mathrm{m}$.

To make possible the operation of a system with many synchronized cameras, a new read-out system is being developed. For each camera the data is processed by fast FPGA electronics (Fig. 3) and possible signals from pellets are extracted. The complete system will handle up to 16 cameras and will efficiently compress the raw data flow from 2 Gbytes/second to a few Mbytes/second, which can be stored and further processed off-line.

The conceptual design for PANDA contains two $400 \mathrm{~mm}$ long vacuum chambers, which can host a full tracking section, one below the pellet generator and another one above the dump. Each section can accommodate four measurement levels (Fig. 4). 


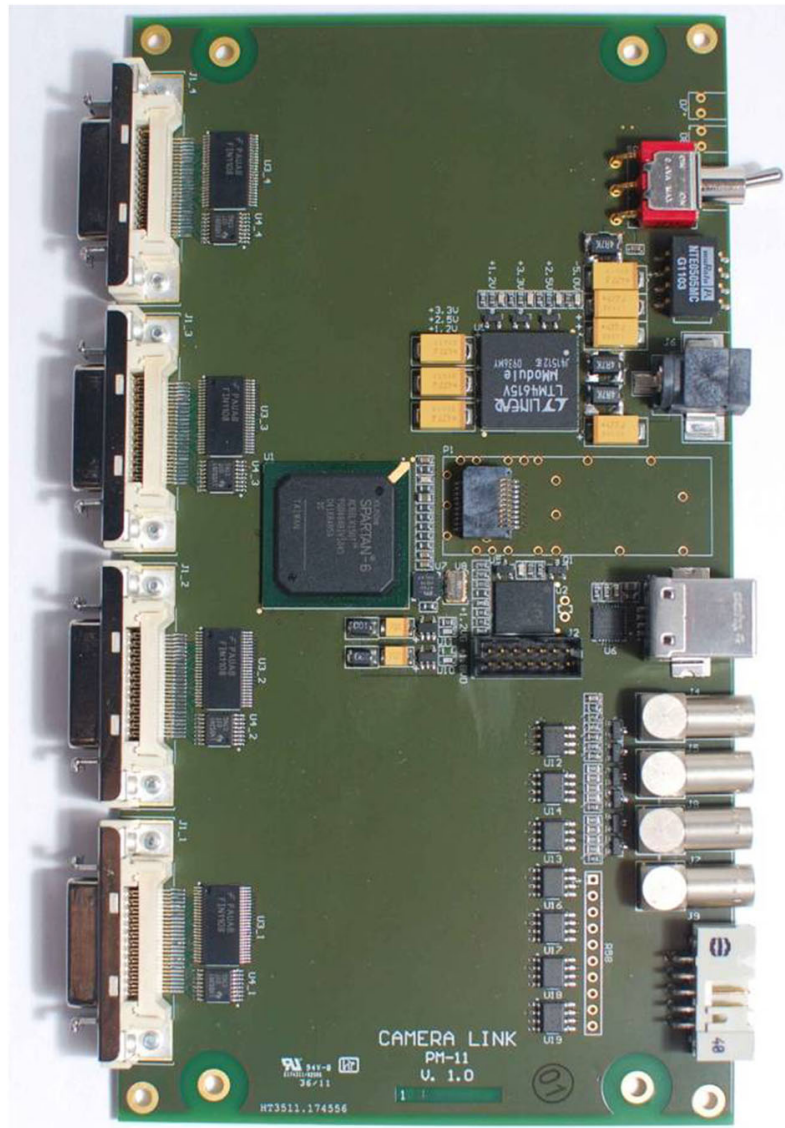

Fig. 3 Prototype FPGA card for readout of four cameras

The design studies include detailed simulations [2] of the measurements of pellet position and time. Various aspects of the measurement, like illumination, optical imperfections and electronics noise are taken into account. Parameters in the simulations are the pellet physical size, brightness, various distortions due to optical effects, camera pixels size and structure of the camera cycle (period and exposure time). Realistic parameter distributions are taken from UPTS experiments. The results of the simulations shows that a resolution in the transverse coordinates of $\sigma \approx 70 \mu \mathrm{m}$ is obtained when using information from the upper tracking section only. For standard conditions, the resolution in the vertical direction depends mainly on the accuracy in the velocity determination which in turn depends on the time resolution from the cameras and the distance between first and last level used in the measurement. The measurement levels in between, improve the resolution slightly $(\approx 30 \%)$ and results in a vertical resolution of $\sigma \approx 0.8 \mathrm{~mm}$. The lower tracking section might improve the resolution (mainly vertically) but is also important for tuning and monitoring of the tracking performance.

The tracking efficiency is defined as the probability that the tracking system gives the correct number of pellets present in the interaction region at a given time. From the results of the simulation studies one can deduce that for the reference conditions about $70 \%$ of the 


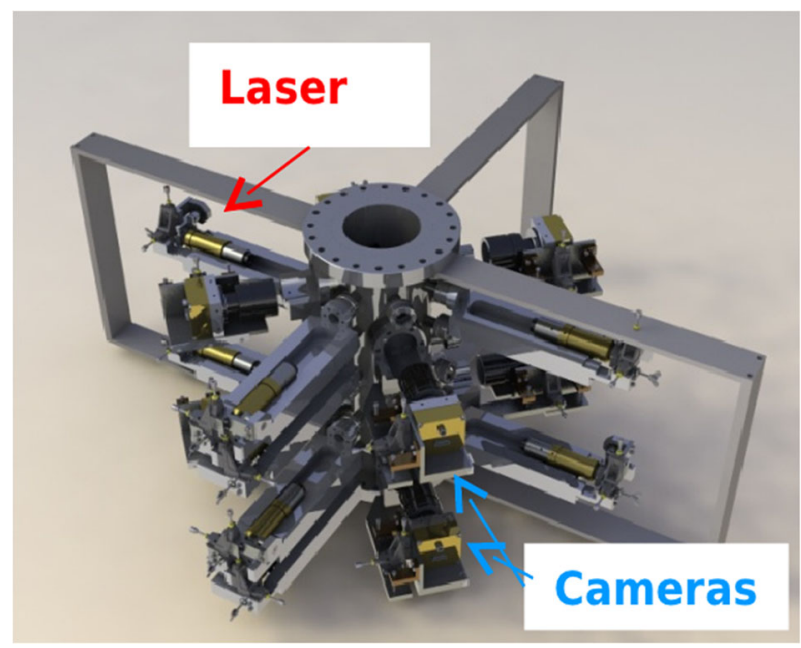

Fig. 4 Conceptual design of one tracking section

hadronic events (i.e. when pellets are in the beam region) would have correct information from the tracking system and for about $50 \%$ of the events there would be unambiguous position information. This fulfills the requirements for PANDA.

Acknowledgments The project is supported by Forschungszentrum Jülich COSY-FFE, European Commission Framework Program 7, Swedish Research Council and Foundation for Polish Science (MPD program) and Polish National Science Center.

Open Access This article is distributed under the terms of the Creative Commons Attribution License which permits any use, distribution, and reproduction in any medium, provided the original author(s) and the source are credited.

\section{References}

1. Calén, H., et al.: Forschungszentrum Jülich IKP Annual Report (2011)

2. Pyszniak, A., et al.: Simulation studies for design of pellet tracking systems, Project report. http://www. physics.uu.se/np/panda/pub/ (2013) 